\title{
Isospin breaking in 2+1 flavor QCD+QED
}

\author{
Ran Zhou*† \\ Physics Department, University of Connecticut, Storrs, CT 06269-3046, USA \\ E-mail: zhouran@phys.uconn.edu
}

\section{Shunpei Uno}

Department of Physics, Nagoya University, Nagoya 464-8602, Japan

E-mail: unodeken.phys.nagoya-u.ac.jp

\begin{abstract}
The mass splittings in the pseudoscalar mesons are studied by combining $2+1$ flavor domain wall fermion gauge configurations, generated by the RBC/UKQCD collaborations, and quenched, non-compact, lattice QED configurations. We extract the QED low energy constants in SU(3) partially quenched chiral perturbation theory, up to next-to-leading order, and determine the nondegenerate $\mathrm{u}, \mathrm{d}$ and $\mathrm{s}$ quark masses. Systematic uncertainties due to chiral extrapolations are discussed. The chiral symmetry breaking of domain wall fermions on the lattice and its effect in partially quenched chiral perturbation theory, including QED effects, is also investigated.
\end{abstract}

The XXVII International Symposium on Lattice Field Theory - LAT2009

July 26-312009

Peking University, Beijing, China

\footnotetext{
* Speaker.

${ }^{\dagger}$ We thank Riken, Brookhaven National Laboratory and the U.S. Department of Energy for providing the facilities and hospitality where this work was done.
} 


\section{Introduction}

The breaking of the strong isospin symmetry exists widely in hadronic physics. For example, the charged pion/kaon and neutral pion/kaon form a strong isospin multiplet. Their masses would be identical if strong isospin symmetry is preserved exactly. But we observe $m_{\pi^{+}}^{2}-m_{\pi^{0}}^{2} \simeq m_{K^{+}}^{2}-$ $m_{K^{0}}^{2} \neq 0$, which means this symmetry is broken in nature. The mass splitting in the hadron spectrum is due to different mass and charge of the fundamental quarks which compose the pion(kaon). The mass splitting in the hadron system can be used as a signature to detect the effect of QED interactions in QCD phenomena and has been studied by the lattice QCD community in recent years $[1,2,3]$. It also provides a way to determine the non-degenerate $\mathrm{u}, \mathrm{d}$ quark masses, which can not be made from numerical simulations including QCD interaction alone. The mass of the lightest quark, as a fundamental parameter of the Standard Model, is related to the strong CP problem and other important questions in high energy physics.

The mass of the pseudoscalar meson is determined by both QCD and QED dynamics. It is difficult to calculate the pseudoscalar mass spectrum theoretically, because of the strong coupling in the QCD sector, which makes perturbation theory invalid. Lattice QCD, on the other hand, is suitable tool to study hadronic physics non-perturbatively. We employ $2+1$ flavor configurations generated by RBC/UKQCD collaborations $[4,5,6]$ in this work, which include not only two degenerate light quarks, but also a heavy strange quark in the sea quark sector. Current lattice simulations can determine the pseudoscalar meson mass to roughly the $1 \%$ level(statistical error only). With the help of the average of $\pm e$ trick [7], we can determine the mass-squared difference, $\delta m^{2}=m^{2}(\mathrm{QCD}+\mathrm{QED})-m^{2}(\mathrm{QCD})$, to even higher accuracy. All of these enable us to build the connection between numerical simulation and theoretical description. For example, our result can be used to fit the Low Energy Constants (LEC's) in QCD+QED Partially Quenched Chiral Perturbation Theory (PQ $\chi \mathrm{PT})$, which are difficult to obtain from experiment alone.

Preliminary results have been reported in $[8,9]$. In this work, we present a new treatment of the violation of chiral symmetry and its further influence on the LEC's and quark mass. We first give the PQ $\chi$ PT description of the pseudoscalar meson mass in Sec. 2. Explicit chiral symmetry breaking in the lattice regularization is discussed in Sec. 3. The fit of QED LEC's and masses are shown in Sec. 4. The summary and plan for future work is given in Sec. $5 .^{1}$

\section{QCD+QED Partially Quenched Chiral Perturbation Theory}

Chiral Perturbation Theory $(\chi \mathrm{PT})$ is a low energy effective theory of QCD based on chiral symmetry. QCD+QED Partially Quenched Chiral Perturbation Theory $(\mathrm{PQ} \chi \mathrm{PT})$ gives the contribution of the sea quark and valance quark to the pseudoscalar meson mass separately, which gives us more freedom to choose the mass of the sea and valance quarks in our lattice simulation. Suppose we have a kaon-like meson which is composed of $\mathrm{u}$ and s quarks. The mass and the charge of the quarks are $m_{1}, q_{1}$ and $m_{3}, q_{3}$ respectively. $q_{i}=2 e / 3,-e / 3$ for up and down/strange quark. So the square of the mass is [10]:

$$
m^{2}=\chi_{13}+\frac{2 C}{F_{0}^{2}} q_{13}^{2}+\frac{\delta^{(4)}}{F_{0}^{2}}
$$

\footnotetext{
${ }^{1}$ The mass splitting in the nucleon system will be reported separately in the future.
} 
where $\chi_{13}=B_{0}\left(m_{1}+m_{3}\right), q_{13}=\left(q_{1}-q_{3}\right)$, and

$$
\begin{aligned}
\frac{\delta^{(4)}}{F_{0}^{2}}= & {\left[\left(48 L_{6}^{r}-24 L_{4}^{r}\right) \bar{\chi}_{1} \chi_{13}+\left(16 L_{8}^{r}-8 L_{5}^{r}\right) \chi_{13}^{2}-\frac{1}{3} \bar{A}\left(\chi_{m}\right) R_{n 13}^{m} \chi_{13}-\frac{1}{3} \bar{A}\left(\chi_{p}\right) R_{q \pi \eta}^{p} \chi_{13}\right] / F_{0}^{2} } \\
& +\bar{A}\left(\chi_{13}\right) q_{13}^{2}+4 \bar{B}\left(\chi_{\gamma}, \chi_{13}, \chi_{13}\right) q_{13}^{2} \chi_{13}-4 \bar{B}_{1}\left(\chi_{\gamma}, \chi_{13}, \chi_{13}\right) q_{13}^{2} \chi_{13} \\
& +C\left[-48 L_{4}^{r} q_{13}^{2} \bar{\chi}_{1}-16 L_{5}^{r} q_{13}^{2} \chi_{13}+2 \bar{A}\left(\chi_{1 s}\right) q_{1 s} q_{13}-2 \bar{A}\left(\chi_{3 s}\right) q_{3 s} q_{13}\right] / F_{0}^{4} \\
& -Y_{1} 4 \bar{Q}_{2} \chi_{13}+Y_{2} 4\left(q_{1}^{2} \chi_{1}+q_{3}^{2} \chi_{3}\right)+Y_{3} 4 q_{13}^{2} \chi_{13}-Y_{4} 4 q_{1} q_{3} \chi_{13}+Y_{5} 12 q_{13}^{2} \bar{\chi}_{1}
\end{aligned}
$$

The definition of symbols used in formula (2.2) can be found in [10]. $C$ is the $\mathscr{O}\left(e^{2}\right)$ low energy constant (LEC). Our $q_{i}$ is $e q_{i}$ of [10]. Our $\mathscr{O}\left(e^{2} m\right)$ LEC, $Y_{i},(i=1,2, \cdots, 5)$, are written in terms of linear combinations of $K_{j}$ of [10].

\section{Violation of chiral symmetry on the lattice}

Domain wall fermions (DWF) live in five-dimensional space and preserve exact chiral symmetry when the size of the extra dimension, $L_{s}$, goes to infinity. But the size of $L_{s}$ is always finite due to the limitation of the computational cost. For example, we set $L_{s}=16$ and 32 in our work, which means we do not have exact chiral symmetry in our simulation. The violation of chiral symmetry is quantified by a small additive shift to the input quark mass, the residual mass, denoted as $m_{\text {res }}$ [6]. Thus the chiral limit is defined as $m_{f}+m_{\text {res }}=0$, which leads us to replace all of the quark masses in the expression for the pseudoscalar mass in PQ $\chi$ PT by $m_{i}+m_{\text {res }}$ [6].

The analysis above is based on QCD interactions. If QED is included, it works as a perturbation to the residual mass which shifts $m_{\text {mres }}$ by $\mathscr{O}\left(e^{2}\right)$ from its QCD value. Following usual perturbative renormalization arguments, the leading QED effect can be written as:

$$
\Delta m_{\mathrm{res}} \equiv m_{\mathrm{res}}(\mathrm{QCD}+\mathrm{QED})-m_{\mathrm{res}}(\mathrm{QCD})=C_{2}\left(q_{1}^{2}+q_{3}^{2}\right)
$$

where $q_{1}$ and $q_{3}$ are the charges of the quarks composing the pseudoscalar meson. The value of $C_{2}$ depends on $L_{s}$ and vanishes exponentially as $L_{s}$ goes to infinity. Higher order terms can be ignored at the order we are working, $\mathscr{O}\left(e^{2} m\right)$.

We next consider the effect of the residual mass to the pseudoscalar meson mass in the QCD+QED simulation. The QED contribution to $m_{\text {res }}$ comes into the mass-squared of the pseudoscalar meson when combined with the leading order QCD term, $2 B_{0} C_{2}\left(q_{1}^{2}+q_{3}^{2}\right)$. We have two different strategies to count this effect. One is to measure the residual mass in the usual way on both QCD+QED and pure QCD configurations from the Ward-Takahashi Identity to get $\Delta m_{\text {res }}$, and extract $C_{2}$. Then we use $2 B_{0} C_{2}\left(q_{1}^{2}+q_{3}^{2}\right)$ to account for the QED induced violation of chiral symmetry. The other way is to add a term $\delta_{\text {mres }}\left(q_{1}^{2}+q_{3}^{2}\right)$ to the expression of $m^{2}$ of the pseudoscalar meson, which means we leave $2 B_{0} C_{2}$ as new free parameter in PQ $\chi \mathrm{PT}$ formula. We can compare whether $\delta_{\text {mres }}$ is consistent with $2 B_{0} C_{2}$, which is a way to check whether our understanding of the QED violation of chiral symmetry is correct or not.

We test our understanding about the violation of the chiral symmetry on $16^{3}$ lattice configurations with $L_{s}=16$ and 32 . The result is shown in figure 1 . The upper line corresponds to the 


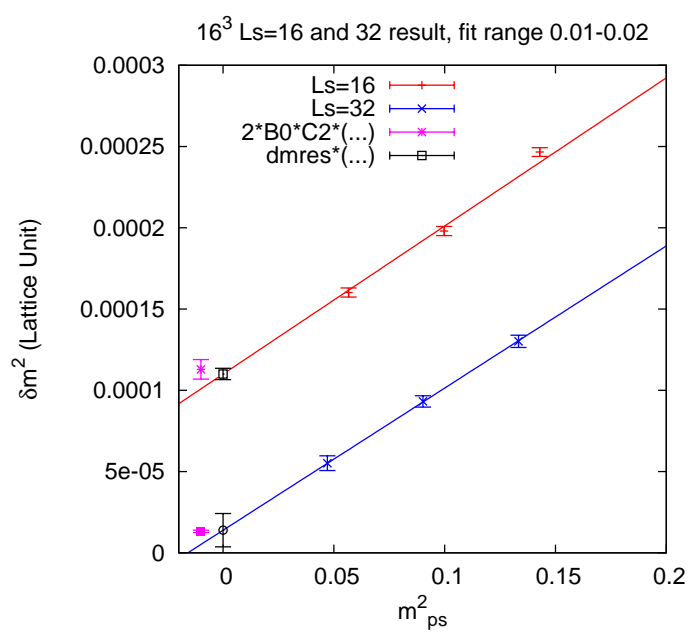

Figure 1: $\delta m^{2}$ of $d \bar{d}$ meson with Ls=16 and 32. dmres(...) means $\delta_{\text {mres }}\left(q_{1}^{2}+q_{3}^{2}\right)$. It's value at $m_{p s}=0$ comes from extrapolation. $2 \mathrm{~B} 0 \mathrm{C} 2(\ldots)$ means $2 B_{0} C_{2}\left(q_{1}^{2}+q_{3}^{2}\right)$. (It is shifted horizontally towards left to make plot clear.) $C_{2}$ is determined from the change of the residual mass by setting $e \neq 0$ and $e=0$. The error bar of this term comes mostly from the error in $B_{0}$.

$L_{s}=16$ case; we measure $\delta m^{2}=m^{2}(\mathrm{QCD}+\mathrm{QED})-m^{2}(\mathrm{QCD})$ of the $d \bar{d}$ meson with $m_{\text {val }}=0.01$, 0.02 and 0.03 and physical charge and extrapolate to $m_{f}+m_{\text {res }}(\mathrm{QCD})=0$ to get $\delta_{\text {mres }}\left(q_{1}^{2}+q_{3}^{2}\right)$. We also determine $C_{2}$ by measuring the change in $m_{\text {res }}$ when $e \neq 0$ and $e=0$. Then the value of $2 B_{0} C_{2}\left(q_{1}^{2}+q_{3}^{2}\right)$ is calculated and shown on the plot (shifted left a little to make the plot clear). The error of $2 B_{0} C_{2}\left(q_{1}^{2}+q_{3}^{2}\right)$ term mostly comes from the error on $B_{0}$. It is clear from figure 1 that these two results are consistent with each other. This consistency is also checked for the $L_{s}=32$ case. Because the violation of the chiral symmetry of DWF is decreased when $L_{s}$ goes to larger value, it is expected that the contribution of the $\delta_{\text {mres }}\left(q_{1}^{2}+q_{3}^{2}\right)$ and $2 B_{0} C_{2}\left(q_{1}^{2}+q_{3}^{2}\right)$ should be suppressed at larger $L_{s}$. It can be seen from the plot that both the $\delta_{\text {mres }}$ and $C_{2}$ terms are decreased by about an order of magnitude at the larger value of $L_{s}$, but their contributions are still consistent within the error bar. All of these confirm the validity of using $\delta_{\text {mres }}\left(q_{1}^{2}+q_{3}^{2}\right)$ to account for the contribution of the violation of the chiral symmetry.

\section{Numerical Result on Low Energy Constant and Quark Mass}

Formula (2.1) can be used to write the mass-squared splitting as:

$$
\delta m^{2}=\delta m^{2}(\text { physical })+\delta \mathrm{m}^{2}(\text { lattice artifact })
$$

where:

$$
\begin{aligned}
\delta m^{2}(\text { physical })= & 2 C q_{13}^{2} / F_{0}^{2}+\bar{A}\left(\chi_{13}\right) q_{13}^{2}+4 \bar{B}\left(\chi_{\gamma}, \chi_{13}, \chi_{13}\right) q_{13}^{2} \chi_{13}-4 \bar{B}_{1}\left(\chi_{\gamma}, \chi_{13}, \chi_{13}\right) q_{13}^{2} \chi_{13} \\
& +C\left[-48 L_{4}^{r} q_{13}^{2} \bar{\chi}_{1}-16 L_{5}^{r} q_{13}^{2} \chi_{13}+2 \bar{A}\left(\chi_{1 s}\right) q_{1 s} q_{13}-2 \bar{A}\left(\chi_{3 s}\right) q_{3 s} q_{13}\right] / F_{0}^{4} \\
& -Y_{1} 4 \bar{Q}_{2} \chi_{13}+Y_{2} 4 q_{p}^{2} \chi_{p}+Y_{3} 4 q_{13}^{2} \chi_{13}-Y_{4} 4 q_{1} q_{3} \chi_{13}+Y_{5} 12 q_{13}^{2} \bar{\chi}_{1}
\end{aligned}
$$

and $\delta m^{2}($ lattice artifact $)=\delta_{\text {mres }}\left(\mathrm{q}_{1}^{2}+\mathrm{q}_{3}^{2}\right)$ 
By fitting the results of $\delta m^{2}$, we can obtain all of the QED LEC's, including $\delta_{\text {mres. }}$ The fit results are shown in figure 2 along with the unitary data points (though all of the partially quenched data points within mass cut were used in the fit). From this figure, we can see that charged meson receives more finite volume corrections than the neutral meson, which is consistent with the theoretical investigation [11]. We perform fits with and without the log terms to probe the effect of the chiral logarithms. $\chi^{2} /$ dof is adequate in either case, except for $24^{3}$ when we include quark masses larger than 0.02 in the fit. One of the reason is that the $\chi^{2} / d o f$ for the pseudoscalar meson mass fit is around 2 at heavier quark mass, which is a little bit large. The other reason is that the quark mass $m_{q}=0.02$ or 0.03 may be too heavy for SU(3) PQ $\chi$ PT [6]. We need to choose the fit range carefully. If the heavier sea quark points are omitted, $\chi^{2}$ becomes reasonable. The main effect of the logs is to significantly reduce the value of the charged meson splitting in the chiral limit (so-called Dashen term). Note, the log terms vanish for the neutral meson splittings at NLO. We put the best result of QED LEC's as we have in Table 1 and compare with the phenomenological value [10]. Our LEC's come from $24^{3}$ lattices fit, which receive less finite volume correction. The fit range is $0.001-0.01^{2}$, which make SU(3) PQ $\chi$ PT to be valid. The log terms are also included in the fit.

\begin{tabular}{|c|c|c|c|c|c|c|c|}
\hline & $10^{6} \mathrm{C}$ & $10^{2} Y_{2}$ & $10^{3} Y_{3}$ & $10^{3} Y_{4}$ & $10^{2} Y_{5}$ & $10^{3} \delta_{\text {mres }}$ & $\chi^{2} /$ dof \\
\hline $24^{3}$ fit & $0.27(19)$ & $1.59(10)$ & $-10.6(7)$ & $9.8(16)$ & $2.00(68)$ & $5.08(9)$ & $2.11(73)$ \\
Ref. [10] & 7.3 & 0.38 & 1.58 & 2.83 & -0.953 & N/A & N/A \\
\hline
\end{tabular}

Table 1: The preliminary result of the QED LEC's from fits of $\delta m^{2}$ and only statistical error is quoted. The QCD LEC's were taken from the SU(3) PQ $\chi$ PT fit by RBC/UKQCD collaborations. All of the QCD and QED LEC's are defined at scale $\mu=1 \mathrm{GeV}$. The last row gives phenomenological estimate in [10].

Next, we determine the physical $\mathrm{u}, \mathrm{d}$, and s quark masses from the QCD LEC's given by the RBC/UKQCD collaborations and the QED LEC's from our fit. The fluctuation in QCD configurations is considered by including the jack knife blocks of QCD LEC's. Since there are three unknown quark masses, we need the experimental value of the mass from three pseudoscalar mesons $\left(\pi^{+}, K^{+}\right.$and $\left.K^{0}\right)$ to fix the quark masses [13]. The mass of the neutral pion is not used, because we don't include the disconnected diagram in our simulation due to the difficulty of computation. The renormalized quark masses are defined as $m_{q}^{\overline{\mathrm{MS}}}=Z_{m}\left(m_{q}+m_{\text {res }}\right)$, where $Z_{m}$ is the matching factor from lattice to $\overline{\mathrm{MS}}(\mathrm{NDR})$ scheme at a scale $\mu=2 \mathrm{GeV}$ obtained via the RI/MOM scheme with the non-perturbative technique ${ }^{3}$. We use the value $Z_{m}=1.656(48)(150)$ from [6]. Table 2 gives the result of the quark masses. The analysis on the $24^{3}$ lattice has less finite volume effect and reasonable $\chi^{2}$ for the LEC's fit for the fit range 0.001-0.01. So we quote the quark mass determined from these parameters as our preliminary result, $m_{u}^{\overline{\mathrm{MS}}}=2.79(37) \mathrm{MeV}, m_{d}^{\overline{\mathrm{MS}}}=4.84(52) \mathrm{MeV}$ and $m_{s}^{\overline{\mathrm{MS}}}=95.9(9.6) \mathrm{MeV}$.

\section{Summary and Plan of Future Work}

Our simulations are based on 2+1 flavor QCD configurations generated by RBC/UKQCD

\footnotetext{
${ }^{2}$ We add $m_{\mathrm{val}}=0.001$ data to our analysis. So our fit result are changed form those reported in [8] as well the result quoted in [9], where only 0.005 and heavier quark masses are used.

${ }^{3}$ Here our $Z_{m}$ is calculated on pure QCD configurations.
} 

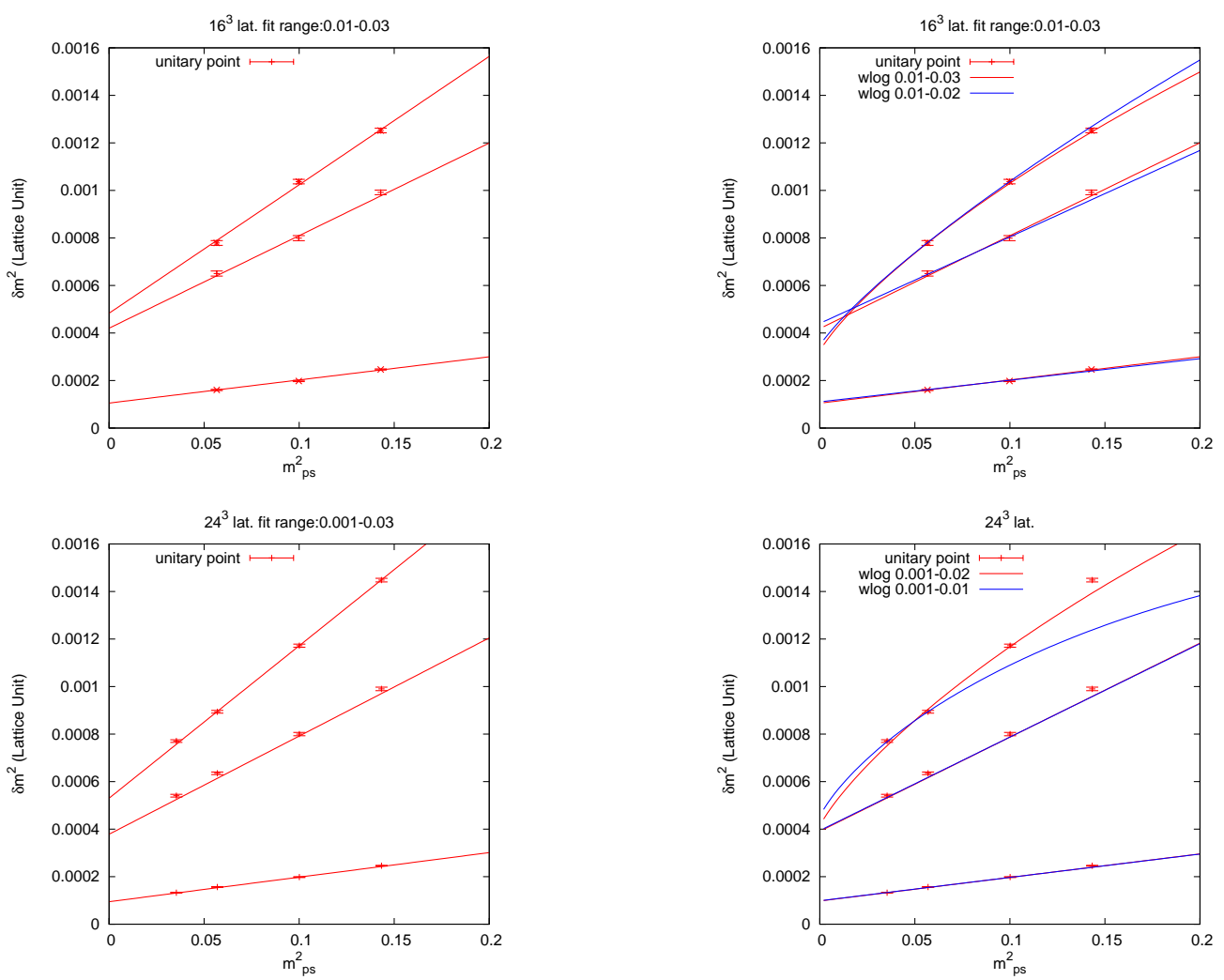

Figure 2: $16^{3}$ linear fit(upper left panel), $16^{3}$ chiral log fit(upper right panel), $24^{3}$ linear fit(lower left panel) and $24^{3}$ chiral $\log$ fit(lower right panel) lattice data points and fit results for the meson mass splitting. Lines correspond to fits with and without chiral logs. The data points in the plot correspond to $u \bar{d}, u \bar{u}$ and $d \bar{d}$ mesons, respectively, from top to bottom.

\begin{tabular}{|c|c|c|c|c|c|c|}
\hline lattice & fit range & $m_{u}$ & $m_{d}$ & $m_{s}$ & $\frac{m_{u}}{m_{d}}$ & $\frac{m_{s}}{m_{u d}}$ \\
\hline $24^{3}$ & $0.001-0.01$ & $2.79(37)$ & $4.84(52)$ & $95.9(9.6)$ & $0.57(1)$ & $25.1(5)$ \\
\hline
\end{tabular}

Table 2: Preliminary results of the $\mathrm{u}, \mathrm{d}$ and $\mathrm{s}$ quark masses determined from pure QCD interaction. The value is in the unit of $\mathrm{MeV}$ and $\overline{\mathrm{MS}}(\mathrm{NDR})$ scheme at renormalization scale $\mu=2 \mathrm{GeV}$. The QCD LEC's were taken from the SU(3) fit by RBC/UKQCD collaborations and QED LEC's are from Table 1. The error quoted here is only statistical error.

collaborations and quenched non-compact QED configurations generated by us. After treating the violation of chiral symmetry carefully, we fit the pseudoscalar meson mass-squared differences using QCD+QED PQ $\chi$ PT to extract the QED LEC's. The LEC's are affected by the volume of the lattice, fit range and the formula used in the fit. We have investigated these systematics, but have not yet quoted errors for them in our preliminary results. The up, down, and strange quark masses are also determined using the LEC with the experimental mass values for pseudoscalar mesons $\left(\pi^{+}\right.$, $K^{+}$and $K^{0}$ ). Our preliminary results for $\overline{\mathrm{MS}}(\mathrm{NDR})$ scheme at the renormalization scale $\mu=2$ $\mathrm{GeV}$ is obtained using LEC's determined on $24^{3}$ lattice and fit range as 0.001-0.01. They are $m_{u}^{\overline{\mathrm{MS}}}=2.79(37) \mathrm{MeV}, m_{d}^{\overline{\mathrm{MS}}}=4.84(52) \mathrm{MeV}$ and $m_{s}^{\overline{\mathrm{MS}}}=95.9(9.6) \mathrm{MeV}$. Errors are statistical only.

In this work, quenched QED configurations were used to account for the EM interaction. The 
systematic error caused by this approximation can be removed by the reweighing method [12]. In addition, the analysis on different lattice spacing will allow to extrapolate to the continuum limit. This will be done on the companion $32^{3}$ lattice ensemble generated by the RBC and UKQCD collaborations (see the talks by Kelly and Mawhinney in these proceedings).

\section{Acknowledgments}

This paper is based on the collaborations with T. Blum, T. Doi, M. Hayakawa, T. Izubuchi and N. Yamada. We thank the US Department of Energy and RIKEN for the support necessary to carry out this research. RZ and TB were supported by US DOE grant DE-FG02-92ER40716. NY is supported by the Grant-in-Aid of the Ministry of Education(No. 20105001, 20105002). TD is supported in part by Grant-in-Aid for JSPS Fellows 21.5985. Computations were carried out on the QCDOC supercomputers at the RIKEN BNL Research Center, BNL, and Columbia University.

\section{References}

[1] A. Duncan, E. Eichten and H. Thacker, Electromagnetic splittings and light quark masses in lattice QCD, Phys. Rev. Lett. 76, (1996) 3894, arXiv:hep-lat/9602005.

[2] S. Beane, K. Orginos and M. Savage Strong-isospin violation in the neutron proton mass difference from fully-dynamical lattice QCD and PQQCD Nucl. Phy. B. 768, (2007) 38-50, arXiv:hep-lat/0605014.

[3] S. Basak et al., Electromagnetic splittings of hadrons from improved staggered quarks in full QCD arXiv:0812.4486 [hep-lat]

[4] D.J. Antonio et. al First results from $2+1$ - flavor domain wall QCD: Mass spectrum, topology change, and chiral symmetry with $L_{S}=8$ Phys. Rev. D. 75, (2007) 114501, arXiv:hep-lat/0612005.

[5] C. Allton et. al $2+1$ flavor domain wall QCD at $(2 \mathrm{fm})^{3}$ lattice: Light meson spectroscopy with $L_{S}=16$ Phys. Rev. D. 76, (2007) 014504, arXiv:hep-lat/0701013.

[6] C. Allton et. al Physical Results from 2+1 Flavor DomainWall QCD and SU(2) Chiral Perturbation Theory arXiv:hep-lat/0804.0703.

[7] T. Blum, T. Doi, M. Hayakawa, T. Izubuchi, and N. Yamada Determination of light quark masses from the electromagnetic splitting of pseudoscalar meson masses computed with two flavors of domain wall fermions Phys. Rev. D. 76, (2007) 114508, arXiv:hep-lat/0708.0484.

[8] R. Zhou, T. Blum, T. Doi , M. Hayakawa, T. Izubuchi, N. Yamada Isospin symmetry breaking effects in the pion and nucleon masses arXiv:0810.1302 [hep-lat]

[9] T.Izubuchi Studies of the QCD and QED effects on Isospin breaking PoS, KAON09 2009,034

[10] J. Bijnens and N. Danielsson Electromagnetic corrections in partially quenched chiral perturbation theory Phys. Rev. D. 75, (2007) 014505, arXiv:hep-lat/0610127.

[11] M. Hayakawa and S. Uno, QED in finite volume and finite size scaling effect on electromagnetic properties of hadrons arXiv:hep-ph/0804.2044.

[12] A. Duncan, E. Eichten and R. Sedgewick, Phys. Rev. D 71, 094509 (2005) [arXiv:hep-lat/0405014].

[13] Amsler et al. Particle Data Group Phy. Lett. B 667, 1 (2008) 